\section{Basic Stereology}

John M. Basgen

University of Minnesota

Basgen@umn.edu

Many us who use microscopes are interested in the internal structure or components of three-dimensional objects. Often we must section these objects to observe these internal components. For many years, microtomes have been used to make physica sections, but in recent years confocal microscopes, MR imaging CT scanners, and even standard optical microscopes have been used to obtain "optical" sections. Two-dimensional images of these different types of sections can be used to extract three-dimensional quantitative information about the objects and their internal components. The sectioning process reduces the observed dimensions of the object and components. With apologies to Rene Magritte, the structure portrayed in Figure 1 is not a three-dimensional glomerulus but a two-dimensional profile of a glomerulus. In most cases, interest is on the structure of the three-dimensional object and not the structure in the two-dimensional image. Thus, care must be taken when obtaining and interpreting data from two-dimensional images [1].

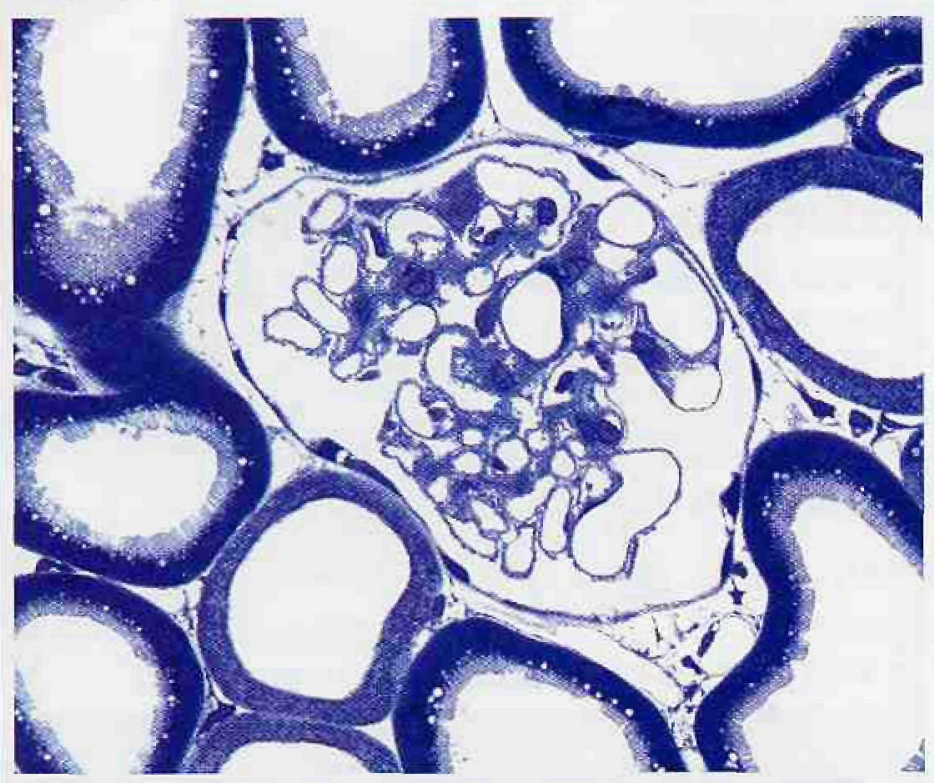

Figure 1. Image of 2-D profile from a 3-D glomerulus

The science of stereology uses information from two-dimensional images of an object to obtain quantitative information about the three-dimensional structure of that object. In recent years, there has been confusion as to exactly what is stereology. Some have confused stereology with morphometry. Morphometry is the measurement of form or structure and thus stereology is a subset of morphometry. Others have confused stereology with the concept of stereoscopy. Stereoscopy uses a pair of two-dimensional images from the same section, obtained at slightly different angles (stereo pairs), and fuses the two images into one three-dimensional image. Three-dimensional reconstruction is another technique that can be used to obtain three-dimensional information from twodimensional samples. After reassembling the 2-D images into a 3-D image, measurements can be made to obtain morphometric information [2]. Adding to the confusion of "What is stereology?" are the different definitions found in various dictionaries. At the International Congress for Stereology held in Melbourne, Australia in November 1999, there was a discussion on how to unify the definition of stereology. The discussion continues to this day.

The word stereology was coined May 11, 1961 by Hans Elias and several colleagues gathered at Feldbert in the Black Forest of Germany. The participants at this meeting were from different fields of study, including biology, material science, and mathematics. They met to discuss how mathematics could help describe the structure of biological and material substances. The International Society for Stereology was founded at that time. As reported in the first issue of the society's bulletin, "stereology deals with a body of methods for the exploration of three-dimensional space, when only two-dimensional sections through bodies or their projections on a surface are available." Elias and his colleagues distinguished this uniaxial viewing method from stereoscopy, a biaxial viewing method [3]. E. R. Weibel formalized the following definition in Stereological Methods: "Stereology is a body of mathematical methods relating three-dimensional parameters defining the structure to two-dimensional measurements obtainable on sections of the structure" [4].

This article briefly describes stereological methods that can used to obtain morphometric information of three-dimensional objects and their components by making appropriate measurments or counts on two-dimensional images of the objects and their components. The basic parameters measured using stereological techniques are: 1) volume, a three-dimensional parameter; 2) area or surface, a two-dimensional parameter; 3 ) length, a one-dimensional parameter; and 4) number, a zero-dimensional parameter. When designing an experiment using stereological methods, it often is advisable to first measure structural densities of the component within the object, then measure the volume of the object, and finally to multiply the component density by the object volume to obtain the actual volume, surface, length, or number of the component.

1. Volume Density $\left[V_{v}(\right.$ Comp/RS $\left.)\right]$ is defined as the ratio of the volume of a component to the volume of the containing object or reference space. When sections are used for measurments, the dimension of the component parameter and the dimension of the reference space have been decreased by one, from the original 3-D structure. Thus, $V_{v}$ (Comp/RS) is obtained from the 2-D image by determining the ratio of the area of the component profile to the area of the reference space profile. The volume density is calculated using the equation, $V_{v}($ Comp/RS $)=$ Area $_{c} /$ Area $_{R S}[5]$. For example, Vv(capillary lumen/glomerulus) could be measured using images such as Figure 1. When measuring volume density, shape of the component and reference space, orientation of the component within the reference space, and sectioning angle are not a problem. This is not the case when measuring surfaces and lengths, as anisotropy possibly may produce biased results.

2. Surface Density [ $S_{\mathrm{v}}$ (Comp/RS)] is defined as the ratio of the surface of a component to the volume of the reference space. Again, in the 2-D image the dimension of the component parameter and reference space is decreased by one. Thus, the surface of interest is reduced to a line and the reference space is reduced to an area. Surface density is measured by determining the amount of this component line length per area of reference space. It is calculated using the equation $\mathrm{S}_{\mathrm{v}}($ Comp/RS $)=\left(2 \times\right.$ Length $\left._{\mathrm{C}}\right) /$ Area $_{\mathrm{RS}}$ [6]. Sv(capillary wall/glomerulus) could be measured using images such as Figure 1. There is no assumption as to the shape of the component but unlike volume density, if there is anisotrophic distribution of the component within the reference space, sections cut at arbitrary sectioning angles may give biased results. To avoid this bias, the vertical section technique must be used [7].

Continued on Page 16 


\section{Sample In - Knowledge Out}

\section{GENERATION} $X$-RAY
The NORAN System SIX Spectral Imaging X-ray system delivers high performance for today's microanalysis applications. Combining highthroughput data acquisition, a complete suite of analytical tools, and high quality reporting, System SIX is designed to get to your results quickly. Sample in - Knowledge out.

The complete package for next generation $\mathrm{x}$-ray microanalysis:

- High-throughput acquisition electronics

- Digital pulse processing and digital beam control

- Single software program for all analysis modes

- Project Data Manager

- Spectral Imaging data archive, extraction and analysis

- COMPASS automatic pure component analysis

- Expert auto peak identification with Spectracheck

- Automated features for optimized data collection and analysis

- One-click formated data reports, Word export

- Industry standard file formats (EMSA, TIFF, CSV)

With more features and functionality than we can list here, the new NORAN System $\mathrm{SIX}$ deserves a closer look.

Call us today at $+1608831-6511$ or visit the NORAN System home page on the web at www.thermo.com/nss.

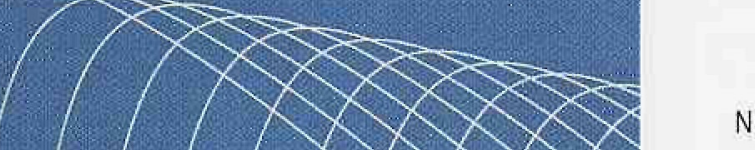




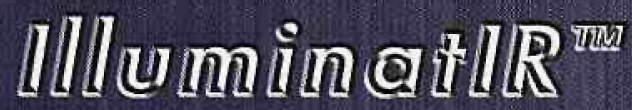

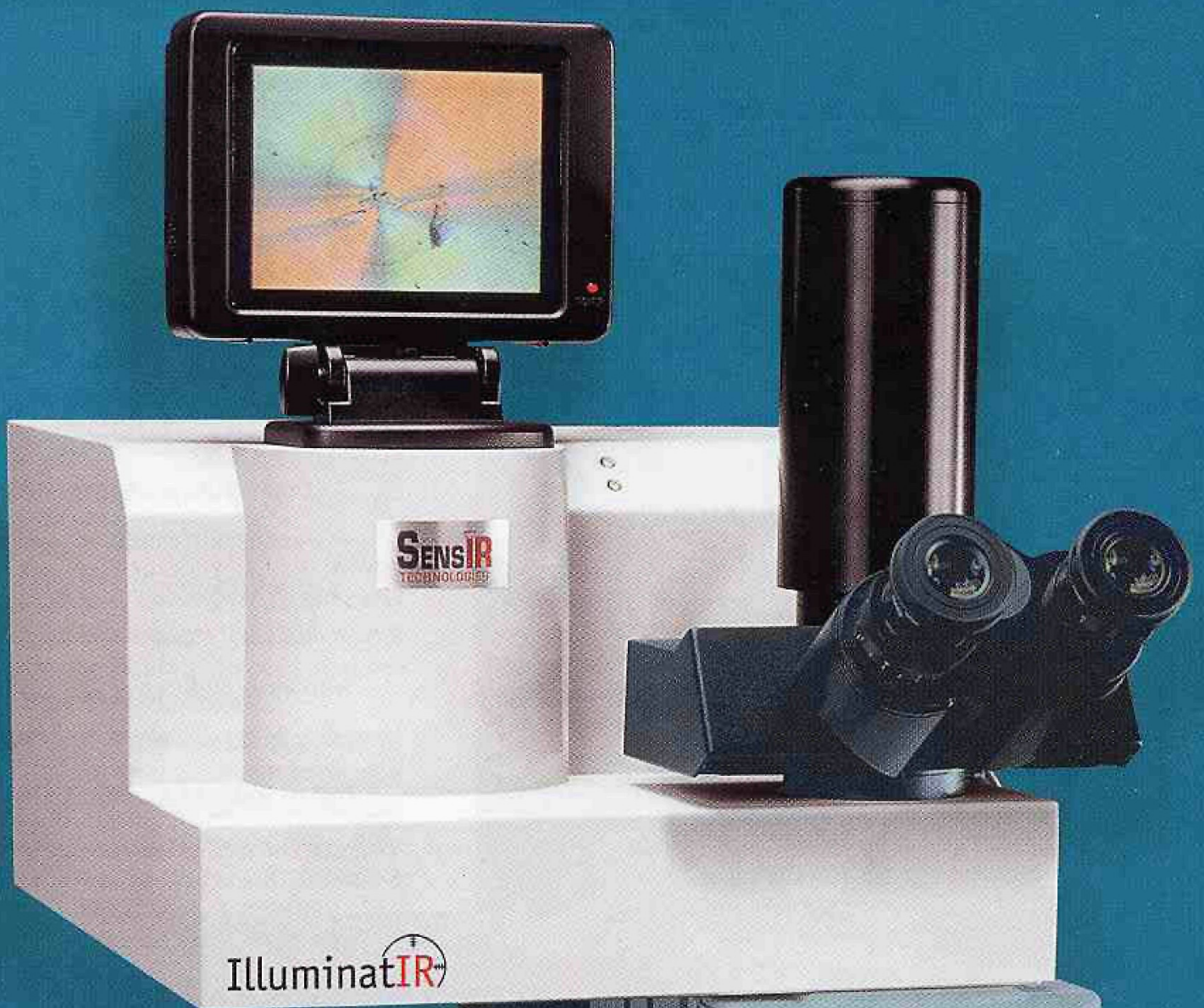

The IlluminatlR is a

miniature FT-IR spectrometer that mounts between the eye piece and the body of an infinity corrected light microscope that requires no additional bench space:
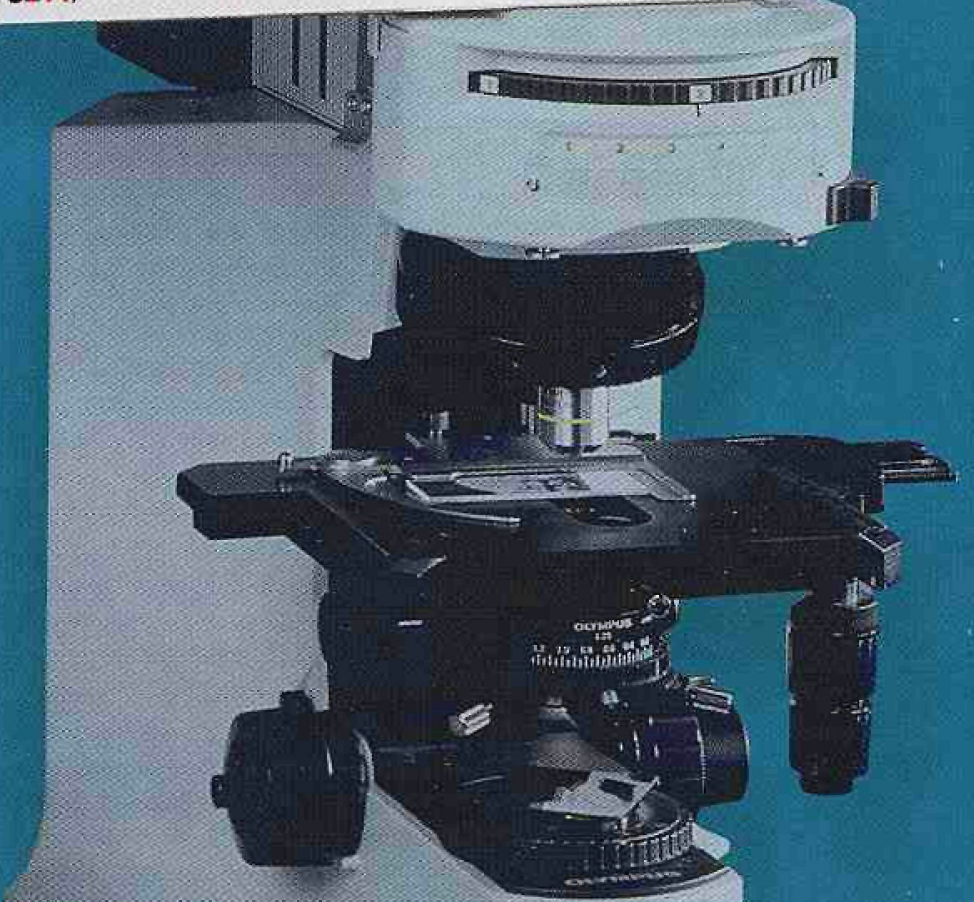


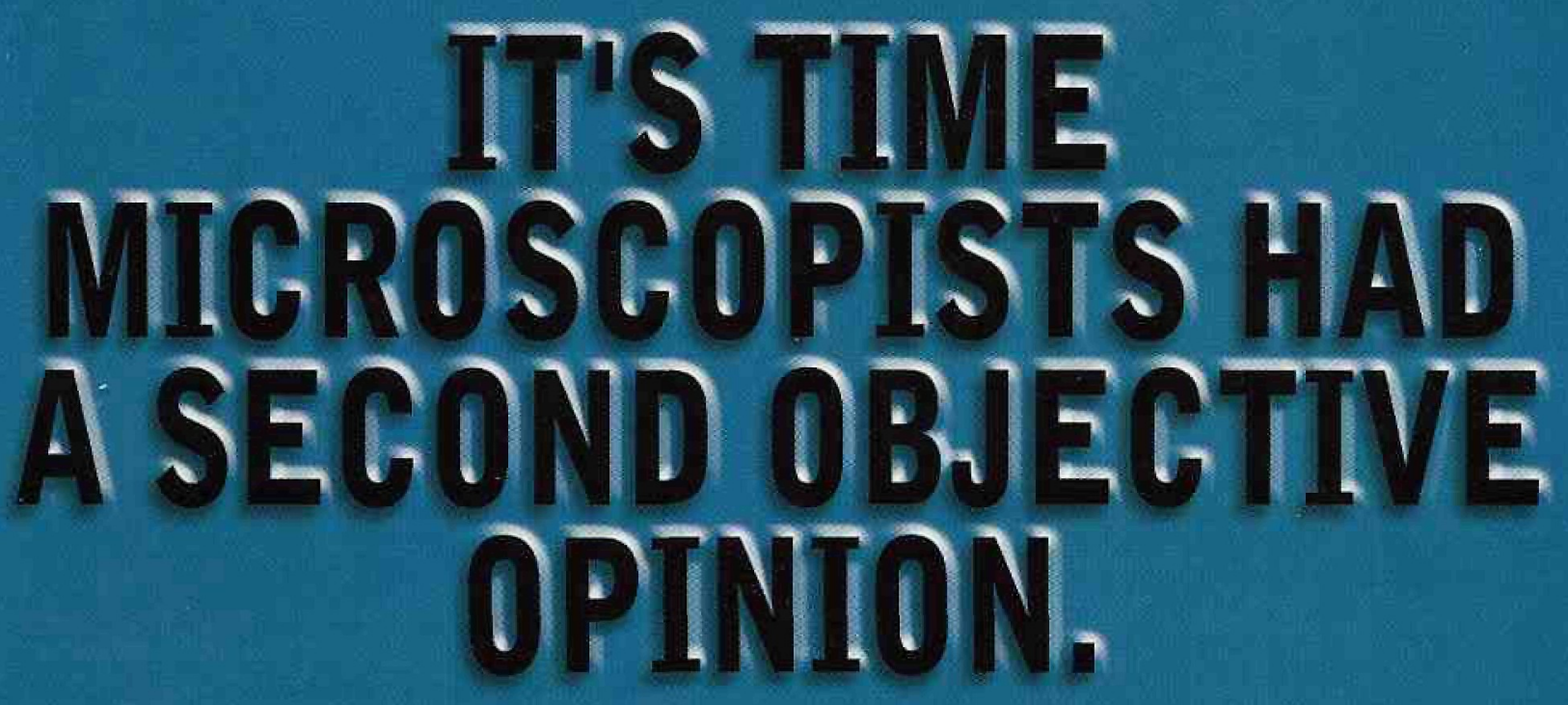

\section{IlluminatIR adds the power of FT-IR analysis to your light microscope}

Introducing the IlluminatIR, the only miniaturized FT-IR system that attaches directly to your favorite light microscope. Add the power of molecular analysis to your visual interpretation for the most accurate and objective results.
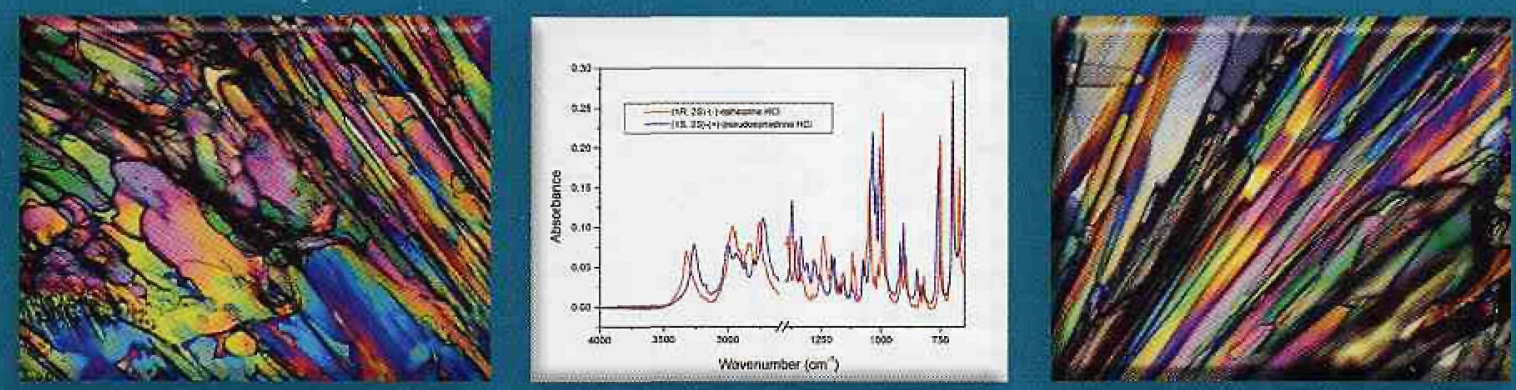

When samplos are visually similar, FT-IR microscopy can show you the difference. The polarized light images of these crystals appeap very similar. However, the results from. FT-IR analysis Ifustratid in the overlaid soectra dearly show the difference between crystal one; which is (-)-Ephedrine HCl, and crystal two which is its isomer ( +$)$-Pseudoephedrine HCl.

Only IlluminatIR:

- provides high quality infrared data and superior visual quality.

- easily attaches to infinity corrected microscopes

- maintains all the capabilities of your microscope,

i.e. polarization, fluorescence, Nomarski or image analysis, etc.

- can run FT-IR analysis simply with the turn of an objective - no need to move the sample

- adds these capabilities without taking up additional bench space

- very easy to use and maintain

The IlluminatIR: it will change the way microscopes are used forever.

\section{MAKE YOUR OWN OBJECTIVE OPINION.}

Call us to arrange a free demonstration of this breakthrough technology. 1-888-473-6747

Or visit our website www.sensir.com/objective

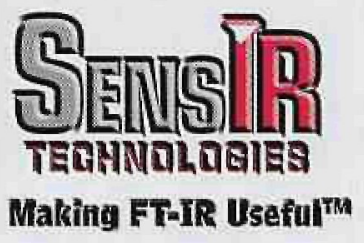


3. Length Density $\left[L_{v}(\mathrm{Comp} / \mathrm{RS})\right]$ is the ratio of the length of a component to the volume of the reference space. Length density has meaning only if the component is much longer than it is wide, such as a fiber or tubule. Again, the dimension of the component parameter and reference space is decreased by one in the image. To measure length density, the number ( 0 dimension) of component profiles is counted and the reference area is measured. The equation to calculate length density is $L_{v}($ Comp/RS $)=\left(2 \times Q_{C}\right) / A^{\prime} a_{R S^{\prime}}$ where $Q_{c}$ is the number of component profiles within the reference area [8]. Lv(capillary/glomerulus) could be measured using images such as Figure 1. If there is orientation of the component within the reference space, often a problem with fibers and tubules, biased results may result unless the vertical slices technique is used to avoid this blas [9].

4. Number Density $\left[N_{v}(\right.$ Comp/RS $\left.)\right]$ is the ratio of the number of component particles per reference space and is the most difficult of the four density parameters to measure. This is because if the dimension of the number parameter is decreased by 1 , a dimension of -1 results. Thus, the number of particles (components) within a three-dimensional structure cannot be obtained from a two-dimensional image!!!. This problem was solved about 15 years ago when the technique known as the disector was first introduced [10]. This technique increases the dimension of the reference sample by using two parallel sections a known distance apart, thus generating a three-dimensional sample. To determine the number of component particles per reference space, a component profile is counted if it is in one section but not in the other. The equation used for calculating this parameter is, $N_{v}($ Comp/RS $)=Q_{C}^{-} /(d x$ Area ${ }_{R S}$ ), where $Q_{C}{ }^{-}$is the number of profiles from components seen in the first section but not seen in the second section. Area ${ }_{\mathrm{RS}}$ is the reference area in the first section and $d$ is the distance between the two sections. Nv(nuclei/glomerulus) could be measured using pairs of images such as Figure 1 that are one or two microns apart. Component shape and orientation are not problems when using the disector. Methods for using optical sections instead of physical sections have been developed [11]

Understanding the meaning of changes of these density measurements can be difficult. If a density parameter is decreased in an experimental group verses a control group, it is not known whether the component is decreased or the volume of the reference space (object) is increased. Therefore, it is advisable to measure the volume of the reference space. Cavalieri, in 1620, published an unbiased method for measuring volume of the reference space independent of shape or orientation. This technique consists of obtaining several sections through the reference space, a known distance apart and measuring the areas of the profiles in the different sections. The volume is calculated using the equation, Volume $\mathrm{RS}_{\mathrm{RS}}$ $=d \times \sum$ Area $_{\mathrm{RS}}$, where $d$ is the distance between the sections and $\sum \mathrm{Area}_{\mathrm{RS}}$ is the sum of the profile areas [12]. Finally, the product of the density measurements and the volume of the reference space results in the appropriate volume, area, length, or number of the component.

Theoretically, stereological methods are reduced to measuring areas and lengths and counting the number of component profiles on two-dimensional images obtained from three-dimensional structures. Practically, there are different ways to make these measurements. If the component can be stained specifically, image analysis systems can quickly determine the appropriate profile areas, component lengths, and component profile number. If the component cannot be stained specifically, the researcher can use image enhancement techniques or semi-manual methods to trace areas and lengths, and count appropriate profiles [13]. These image enhancement and tracing techniques can be quite time consuming. Often a more efficient measuring method is to superimpose an appropriate counting grid of points and/or lines over the image [14]. Grid points falling on profiles are counted to estimate areas, intercepts between grid lines and component lines are counted to estimate lengths, and number of component profiles is counted when determining length and number density. Counting between 100 and 200 points, intercepts, or profiles per animal gives sufficient precision for most biological experiments and takes surprisingly little time [15]. A follow-up article in a future issue will discuss sampling and methods for determining the optimal number of animals per experimental group, blocks per animal, and measurements per animal.

The International Society for Stereology maintains a website (www.stereologysociety.org), which lists information related to workshops and future meetings.

\section{References}

1. Elias H. (1971) Three-dimensional structure identified from single sections. Science 171:993-100

2. Gaunt PN and Gaunt WA. (1978) Three dimensional reconstruction in biology. University Park Press, Baltimore.

3. Elias H. (1962) Stereologia 1:1

4. Weibel ER. (1979) Stereological Methods: Practical Methods for Biological Morphometry Academic Press, New York p. 1.

5. Howard CV and Reed MG. (1998), Unbiased Stereology Three-Dimensional Measurement in Microscopy, Bios Scientific Pub., New York pp. 55-68.

6. Howard CV and Reed MG. (1998), Unbiased Stereology Three-Dimensional Measurement in Microscopy, Bios Scientific Pub., NY pp. 107-108.

7. Baddeley AJ, Gundersen HJG and Cruz-Orive LM. (1986) Estimation of surface area from vertical sections. Journal of Microscopy 142:259-276.

8. Howard CV and Reed MG. (1998), Unbiased Stereology Three-Dimensional Measurement in Microscopy, Blos Scientific Pub., NY pp. 125-128.

9. Gokhale AM. (1993) Utility of the horizontal slice for stereological characterization of linear features. Joumal of Microscopy 170:3-8.

10. Sterio DC. (1984) The unbiased estimation of number and sizes of arbitrary particles using the disector. Journal of Microscopy 134:127-136.

11. Braendgaard H, Evans SM, Howard CV and Gundersen HJG. (1990) The total number of neurons in the human neocortex unbiasedly estimated using optical disectors. Journal of Microscopy 157:285-304.

12. Gundersen HJG and Jensen EB. (1987) The efficiency of sampling in stereology and its prediction. Journal of Microscopy 147:229-263.

13. Russ JC. (1995) The Image Processing Handbook, 2nd Edition, CRC Press, Boca Raton.

14. Basgen JM, Rich SS, Mauer SM and Steffes MW. (1988) On measuring volume density of the glomerular mesangium. Nephron 50:182-186.

15. Gundersen HJG and Osterby R. (1980) Optimizing sampling efficiency of stereological studies in biology: or "do more less well!?. Journal of Microscopy 121:65-73

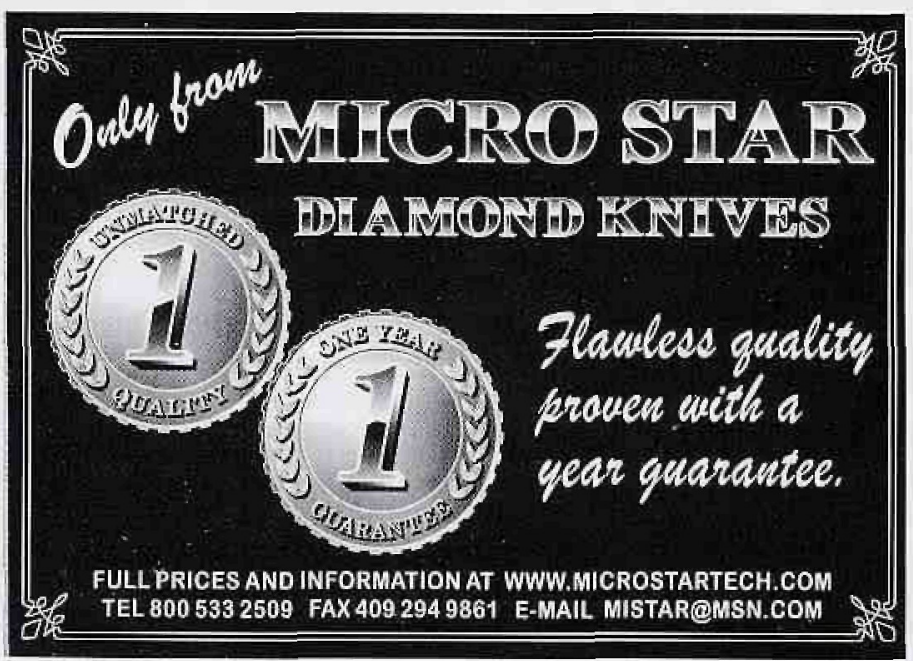




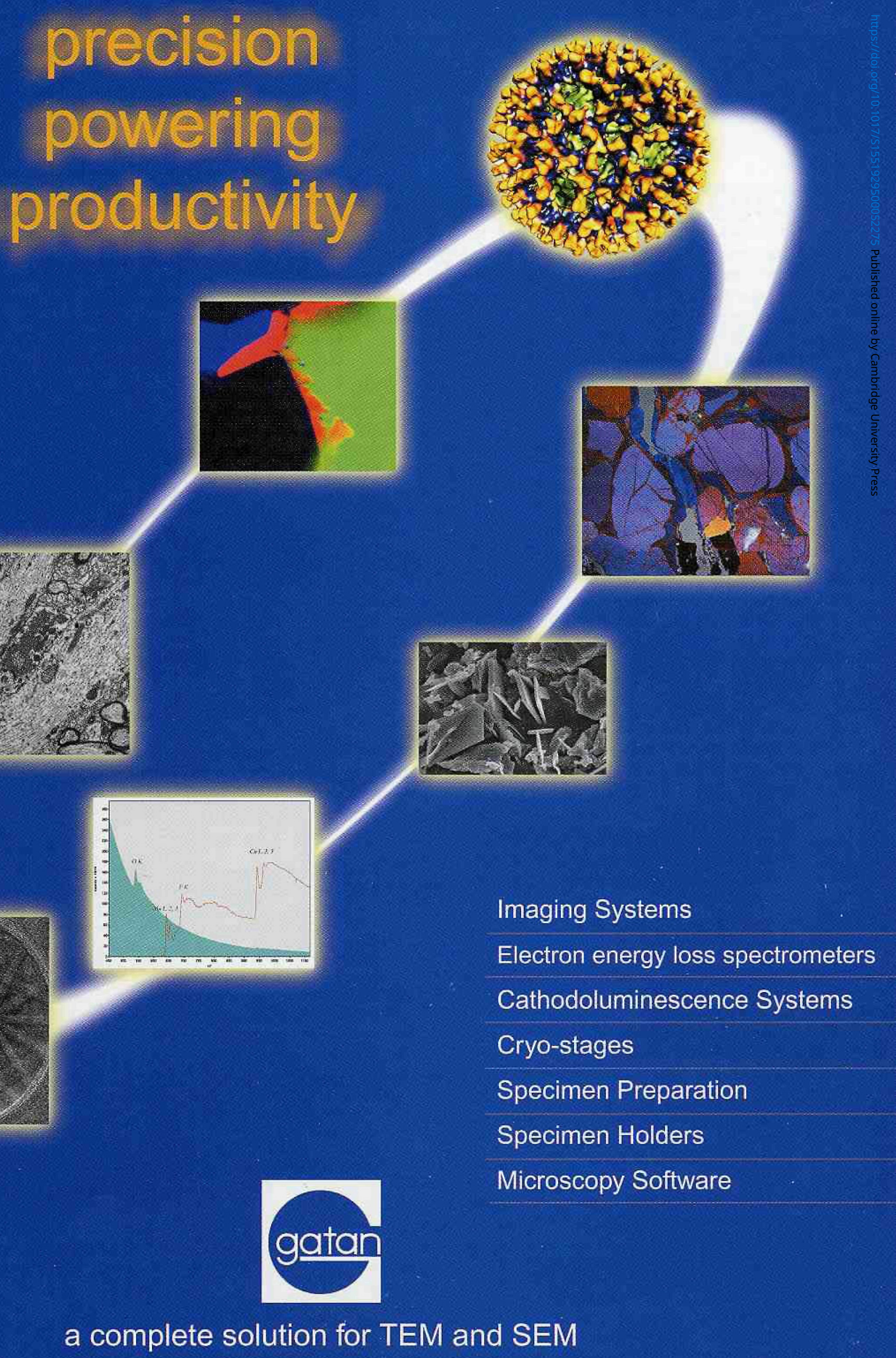

